\title{
Aprendizaje adaptativo en moodle: tres casos prácticos
}

\section{Adaptive learning in moodle: three practical cases}

Dolores Lerís López, Fernando Vea Muniesa, Ángeles Velamazán Gimeno

Departamento de Matemática Aplicada, Universidad de Zaragoza, España. \{dleris, fernavea, mavelama\}@unizar.es

\section{Resumen}

Uno de los retos más importantes que habrá de afrontar la educación es la necesidad de adecuar el proceso formativo a las características del estudiante. Hoy día todavía se señala el deficiente apoyo tecnológico y las escasas prácticas de personalización del aprendizaje. La tecnología educativa que apoya el e-learning ha ido desarrollando dos tipos de plataformas: Sistemas de Gestión del Aprendizaje (LMS) y Sistemas Hipermedia Adaptativos dirigidos a la educación. Ambas líneas de desarrollo están convergiendo, de forma que las nuevas versiones de los LMS incorporan capacidades adaptativas que van permitiendo diseñar cursos individualizados o diferenciados. En este artículo se revisan las funcionalidades adaptativas disponibles en Moodle. Se explica cómo se han puesto en práctica tres casos de diseños instruccionales adaptativos. Además, se constata su eficacia, en términos del aprendizaje logrado por el estudiante, y su eficiencia, al reutilizar materiales de experiencias anteriores.

\section{Palabras Clave:}

Aprendizaje personalizado; Sistemas Adaptativos de Aprendizaje; Moodle; Tecnología Educativa; Casos prácticos; Eficacia.
One of the most important challenges that the education will have to face is the need to adapt the learning process to the student's characteristics. Nowadays it is still noticed the weak technological support and the few personalised learning practices. Two main types of e-learning platforms have been developed for last years: Learning Management Systems (LMS) and Adaptive Educational Hypermedia Systems. Both lines of development are converging so that the new versions of the LMS incorporate adaptive capacities that are allowing to design individualized or differentiated instruction. In this paper the adaptive functionalities available in Moodle are checked. It is explained how to implement three adaptive instructional designs in Moodle. Moreover, it is checked their effectiveness, in terms of the learning achieved by the student, and their efficiency, by reusing materials of previous learning experiences.

\section{Keywords:}

Personalised learning; Adaptive e-Learning Systems; Moodle; Educational Technology; Practical cases; Effectiveness. 


\section{Introducción}

Uno de los retos más importantes que habrá de afrontar la educación es la necesidad de adecuar los procesos de aprendizaje a las necesidades, intereses y conocimientos del estudiante. Johnson, Adams, Cummins y Estrada (2012) señalan el aprendizaje personalizado como el tercer desafío, en orden de importancia, de los diez registrados como más significativos por su impacto en la docencia, en el aprendizaje o en la investigación en el ámbito universitario en los próximos cinco años y afirman: "The demand for personalized learning is not adequately supported by current technology or practices". Aunque la idea de personalizar el aprendizaje no es nueva en el mundo académico, su demanda ha crecido especialmente en la última década, en la que el paradigma educativo centrado en el aprendizaje ha cobrado fuerza. Si el objetivo de este paradigma imperante es que el estudiante alcance los mayores logros posibles, entonces es necesario adaptar la enseñanza a cada uno de ellos considerado como persona individual. Como afirma Miliband (2006): "Personalised learning is the way in which our best schools tailor education to ensure that every pupil achieves the highest standard possible" (p. 24). Más aún, las actuales apuestas educativas, fuera del ámbito puramente académico, como la formación en las empresas o los cursos en línea masivos y abiertos, barajan la adaptación de la formación al usuario como solución a las altas tasas de abandono ( Fidalgo-Blanco, García-Peñalvo y Sein-Echaluce, 2013; Sonwalkar, 2013; Amo, Casany y Alier, 2014; Daniel, Vázquez y Gisbert, 2015; FidalgoBlanco, Sein-Echaluce y García-Peñalvo, 2015).

Para alcanzar y consolidar la personalización del aprendizaje, la individualización o la diferenciación de las experiencias de aprendizaje (Calderero, Aguirre, Castellanos, Peris y Perochena, 2014), en entornos educativos formales con gran número de estudiantes, es imprescindible el apoyo tecnológico y, desde luego, suficientes prácticas educativas que avalen su viabilidad y efectividad.

El desarrollo tecnológico ha permitido pasar de las máquinas de enseñar, como la de Skinner (1958) a los sistemas electrónicos que apoyan el aprendizaje. Estos últimos han avanzado en dos direcciones. Por un lado, los sistemas de gestión del aprendizaje LMS (acrónimo de Learning Management Systems) y, por otro, los hipermedia adaptativos AHS (acrónimo de Adaptive Hypermedia Systems) para la educación.

Los LMS disponen de funcionalidades que apoyan la gestión de cursos, de usuarios, de grupos, de calificaciones, etc. y también permiten la gestión de contenidos (GarcíaPeñalvo y Seoane-Pardo, 2015). En el contexto universitario español, los responsables de las políticas académicas de las universidades 
han apoyado la puesta en marcha de campus virtuales de docencia, basados en LMS como Moodle. Ese esfuerzo ha redundado en la incorporación paulatina y constante de muchos profesores universitarios al sistema del aprendizaje semipresencial o blendedlearning.

Los AHS para la educación son sistemas capaces de registrar las acciones del estudiante, de interpretarlas según el modelo de usuario asociado y, en consecuencia, de adecuar el proceso de aprendizaje al individuo. Así pues, el sistema adapta su comportamiento a cada persona, es decir, personaliza el aprendizaje. La difusión y el uso de los AHS en la educación son, hoy día, muy reducidos, principalmente por dos motivos. Uno de ellos se encuentra en el paradigma predominante en la investigación de los sistemas adaptativos, según el cual un equipo de expertos desarrolla un software que soporte la adaptación al usuario y ellos mismos lo prueban y evalúan (Brusilovsky, Knapp y Gamper, 2006). Habitualmente, el software desarrollado requiere que el profesorado, que son los autores del diseño instruccional y de las actividades, dedique gran esfuerzo para poder utilizarlo. El otro motivo podría ser que los sistemas e-learning con adaptación inteligente necesitan disponer de un número suficiente de contenidos adecuadamente etiquetados, para que el sistema los organice y presente automáticamente al usuario en función de los datos que de él va recogiendo (Rey-López et al., 2008). Esta condición exige de nuevo un notable esfuerzo de autoría, es decir, de creación y etiquetado de esos contenidos inteligentes; junto con productos tecnológicos que faciliten su localización y utilización (Fidalgo, Sein-Echaluce, Lerís y Castañeda 2013; Fidalgo-Blanco, GarciaPeñalvo, Sein-Echaluce y Conde-González, 2014).

Los autores de este artículo trabajamos en el aprendizaje adaptativo desde nuestra perspectiva de profesores, que lo practican en la docencia universitaria. Por ello, hemos observado con agrado el interés en dotar a los LMS, en particular a Moodle, con características adaptativas. Que el LMS disponible en el campus virtual de la universidad ofrezca posibilidades adaptativas es, de hecho, un paso decisivo hacia el objetivo de la personalización del aprendizaje.

En el artículo se examinan las posibilidades adaptativas de la versión 2.4 de Moodle. Además, se presentan tres casos prácticos de diseños instruccionales utilizando las capacidades adaptativas de dicha versión. 


\section{Contexto y diseños adaptativos}

Moodle (acrónimo de Modular Object Oriented Dynamic Learning Environment) es un LMS de código abierto de gran versatilidad, pues permite generar cursos con muy diferentes configuraciones. Moodle es utilizado tanto en contextos formativos puramente virtuales como en la formación presencial, en la que es un valioso complemento.

Además de las posibilidades para administrar cursos, usuarios, grupos o calificaciones, Moodle permite incorporar recursos y actividades de muy diversa índole. Un curso Moodle puede contener recursos pasivos, como los archivos o las páginas web, o activos/colaborativos como las wikis, los glosarios o las bases de datos; herramientas de comunicación asíncrona, como los foros o la mensajería interna, o de comunicación sincrónica, como los chats; herramientas que soportan la auto-evaluación, la co-evaluación o la evaluación por rúbricas, como los cuestionarios, los talleres o las tareas; etc.

Las posibilidades adaptativas de Moodle aparecen esencialmente en dos direcciones. Se podría decir que una es intra-actividad y otra es inter-actividades. La primera significa que la adaptación al usuario es una posibilidad de la configuración interna de la actividad; mientras que la adaptatividad inter-actividades supone el establecimiento de reglas o relaciones de dependencia entre diferentes actividades.

Algunas actividades de Moodle tienen cierta capacidad adaptativa propia, como objetos de aprendizaje independientes del resto de recursos o actividades de un curso Moodle. Por ejemplo, esta adaptatividad intra-actividad está disponible en los módulos de lecciones, cuestionarios, foros y SCORM (acrónimo de Sharable Content Object Reference Model).

- Las lecciones de Moodle son actividades de aprendizaje que proporcionan contenidos de forma individualizada siguiendo una programación ramificada. Las lecciones están formadas por páginas puramente de contenidos o con una pregunta. En las primeras, se ofrece al estudiante la posibilidad de elegir la siguiente página que quiere ver $y$, en las segundas, la respuesta dada a la pregunta que contiene determina la próxima página que el estudiante va a ver. La navegación en una lección puede ser lineal o tan compleja como el formador estime necesario.

Incluso es posible añadir cierta adaptatividad inter-lecciones, pues se pueden establecer como prerrequisitos, de acceso a una lección, algunos parámetros de una lección previa como el tiempo empleado, el haberla finalizado o la calificación obtenida. De esta forma, se puede generar un camino lineal a través de diferentes lecciones de un mismo curso.

También se puede configurar una lección de modo que, una vez finalizada, el sistema presente el enlace a otra actividad del curso. 
De este modo el diseñador del curso está guiando al estudiante indicándole cuál es la siguiente actividad que se le recomienda realizar.

- Los cuestionarios de Moodle son actividades en las que se ofrece al estudiante una lista de preguntas cuya respuesta conduce a una calificación. La adaptatividad de un cuestionario se establece al configurar la visualización de la retroalimentación incluida en las preguntas y sus opciones de respuesta, así como al seleccionar el comportamiento del cuestionario con interactividad para que muestre, si fuera necesario, las pistas añadidas a las preguntas. Se trata, pues, de una realimentación individualizada, inmediata y automática.

- Los foros de Moodle son actividades de comunicación asíncrona, que pueden ser configurados de diferentes maneras; una de ellas permite que el foro se separe o diferencie por grupos de usuarios. En el tipo de foro de Pregunta y Respuesta (abreviadamente, PyR), el debate se inicia cuando el tutor envía un mensaje con una pregunta a la que los estudiantes responden. Un foro PyR tiene una pequeña característica adaptativa, ya que requiere que un estudiante conteste una vez, antes de ver las respuestas de los demás. Tras enviar su respuesta inicial, los estudiantes ya pueden ver y contestar a los mensajes de sus compañeros.

- El módulo SCORM de Moodle permite cargar cualquier paquete estándar
SCORM de objetos de aprendizaje y convertirlo en parte de un curso. Un paquete SCORM es un bloque de material web (páginas web, gráficos, programas Javascript, presentaciones Flash, etc.). Vélez, Baldiris, Nassiff y Fabregat (2008) muestran cómo generar un SCORM adaptativo, que admite ser integrado en la plataforma Moodle.

La solución adaptativa de los módulos de Moodle, mencionada en los párrafos anteriores, consiste en una personalización local del proceso de aprendizaje, es decir, tan solo se adapta la respuesta del sistema dentro de una actividad. La generación de un curso adaptativo completo es un asunto más ambicioso y más complejo, tanto desde el punto de vista técnico como práctico, pues necesita que el sistema admita un modelo de usuario y permita la definición de reglas de transición entre los conceptos del curso.

Para convertir Moodle en un sistema adaptativo se han ensayado distintas soluciones. Una de ellas ha consistido en comunicar/integrar Moodle con un Sistema Hipermedia Adaptativo. Por ejemplo,

- Tiarnaigh (2005) propuso y ensayó la integración de Moodle con APeLS, acrónimo de Adaptive Personalized eLearning Service (Conlan, Wade, Gargan, Hockemeyer y Albert 2002).

- La especificación IMS Learning Design, que admite diseños adaptativos (Berlanga y García-Peñalvo, 2004, 2005, 2008) fue objeto de un intento de interoperabilidad con Moodle (Burgos, Tattersall, 
Dougiamas, Vogten y Koper, 2007).

- Mucho más ambicioso es el proyecto GRAPPLE (Generic Responsive Adaptive Personalized Learning Environment), 2008-2011, en el que se pretende que un entorno adaptativo de aprendizaje llegue a ser una componente de los LMS (Bra et al., 2013).

Otro tipo de soluciones, como la ofrecida por el Centro de Innovación para la Sociedad de la Información (CICEI), ha consistido en modificar el código de Moodle, para así lograr configurar recursos o actividades en función de que se cumplan determinadas condiciones en otros. Su primera versión fue presentada a la comunidad Moodle en 2005. A pesar de las extraordinarias posibilidades adaptativas de estos multi-condicionales del CICEI (Lerís y Sein-Echaluce, 2011), las sucesivas versiones estándar de Moodle 2 han ido incorporando soluciones adaptativas más limitadas.

Con la llegada de la versión 2 de Moodle aparece la posibilidad de adaptatividad inter-actividades, pues se dota a Moodle de algunas capacidades para adaptar la navegación y presentación de contenidos en función de las acciones del usuario. Se podría decir que la idea de la dependencia entre lecciones, disponible en versiones anteriores, se ha extendido a otras actividades y se ha ampliado en cuanto a tipos de condiciones de dependencia.

En Moodle 2 se puede parametrizar una actividad o un recurso de forma que se condicione su acceso bien por fechas, bien por el perfil Moodle del usuario e incluso por

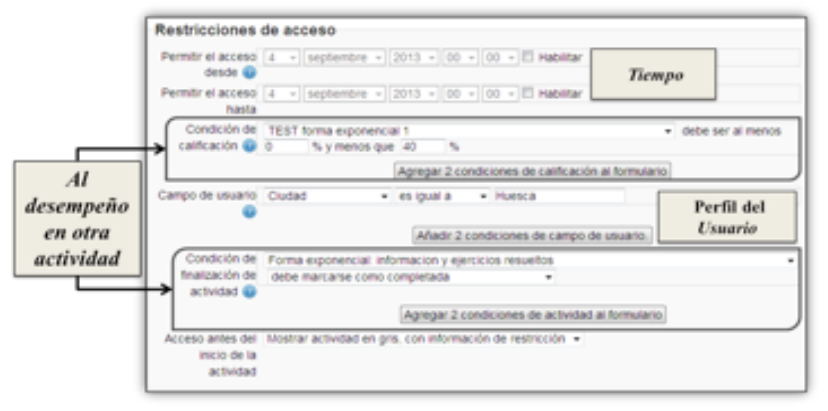

Figura 1. Prerrequisitos de acceso a un contenido Moodle (imagen de la versión 2.4).

el desempeño (calificación, finalización) del estudiante en otras actividades o recursos del mismo curso Moodle. El diseñador del curso encuentra todas esas opciones en el apartado Restricciones de acceso del formulario de ajustes de cada recurso o actividad (véase la figura 1).

Las condiciones de calificación posibilitan restringir el acceso en función de la obtenida en otra u otras actividades calificables. En el campo de condiciones de finalización de otras actividades, se pueden establecer prerrequisitos de haber sido finalizada una actividad o de haber sido aprobada o sus respectivas negaciones. El valor de finalización se ajusta en el propio formulario de configuración de una actividad; sin embargo, el significado de calificación aprobada ha de ajustarse en el correspondiente ítem de calificación del libro de calificaciones.

También hay un parámetro que controla si el contenido ha de estar visible al usuario; se puede optar bien por ocultarse, en tanto no se cumplen las condiciones exigidas para su acceso, o bien por mostrarse en gris, con la información de las condiciones que se han de cumplir para que sea accesible. En las versiones de Moodle anteriores a la 2.7, todas 
las condiciones impuestas se conectaban únicamente con el operador lógico "and", lo cual suponía una importante dificultad para programar diferentes itinerarios de aprendizaje.

En resumen, cada actividad Moodle almacena los prerrequisitos para su acceso, el modo de visualización y el significado de haber sido finalizada. Todos ellos son parámetros que están involucrados en la generación de cursos adaptados al usuario.

Las posibilidades adaptativas del sistema e-learning, que se desee utilizar, determinan, en general, el enfoque para crear cursos adaptativos. Se han identificado tres tipos principales de enfoques para crear cursos adaptativos (Vassileva, 2010; Bontchev y Vassileva, 2012).

El enfoque más sencillo y más fácil para crear cursos adaptativos es generarlo con un contenido diferente para cada estudiante o grupo de ellos. Por ejemplo, DespotovićZrakić, Marković, Bogdanović, Barać y Krčo (2012) recogen datos de los estudiantes, sus estilos de aprendizaje y su comportamiento en un precurso Moodle, que son tratados, utilizando técnicas de minería de datos, hasta conseguir clasificarlos en tres grupos (cluster). A cada uno de ellos se le ofrecía $\mathrm{su}$ particular curso en Moodle. En este enfoque puede resultar muy útil contar con los agrupamientos de Moodle, ya que permiten que el profesor asigne contenidos específicos, o incluso secciones completas, a los estudiantes incluidos en un determinado agrupamiento (Lerís y Sein-Echaluce, 2011).
Otro enfoque utiliza una red de conceptos conectados entre sí. Habitualmente, estas relaciones definen el orden de los conceptos para ser visitados por un aprendiz. Y la tercera forma de crear un curso adaptativo consiste en el establecimiento de reglas para la transición de un concepto a otro o de una página a otra (Vassileva, 2010; Bontchev y Vassileva, 2012).

Ese último enfoque es el utilizado por el Grupo de Innovación e Investigación en Docencia con el uso de las Tecnologías de la Información y la Comunicación (GIDTIC) en las experiencias de un curso de apoyo de matemáticas (Castelló, Lerís, Martínez y Sein-Echaluce, 2010) y un curso sobre trigonometría (Sein-Echaluce, Lerís y Fidalgo, 2011), soportado por Moodle 1.8 con los multi-condicionales del CICEI, y en los casos que se exponen en las siguientes secciones de este artículo con el apoyo de Moodle 2.4. Tanto en las experiencias previas como en los casos prácticos descritos en este trabajo, las reglas de transición de un concepto a otro forman parte de los parámetros asociados a una actividad y han de ser especificados en el propio curso, con las dificultades de autoría que eso produce en términos de esfuerzo y de programación lógica.

Es un hecho que la capacidad adaptativa de Moodle ha ido mejorando en las sucesivas versiones de Moodle 2. Por ello, entendemos que es oportuno poner en marcha cursos, o unidades de aprendizaje, adaptativos sencillos y útiles en el contexto educativo formal, que demuestren su eficacia y eficiencia. 
En los siguientes apartados se describen cada adaptativas, puestos en práctica en la una de las tres experiencias educativas, bien Universidad de Zaragoza durante el semestre de cursos o bien de unidades de aprendizaje de primavera de 2013.

\section{Contexto y diseños adaptativos}

El objetivo general de las experiencias fue mejorar el proceso y los resultados del aprendizaje de nuestros alumnos. Para ello, se diseñaron procesos de aprendizaje adaptados a las características de cada estudiante en tres asignaturas del Área de Matemáticas, que fueron experimentados en el segundo semestre del curso académico 2012-2013.

Los correspondientes diseños instruccionales fueron implementados en Moodle, plataforma que da soporte al campus virtual de la Universidad de Zaragoza (en adelante, UZ). Se utilizaron las capacidades adaptativas disponibles en la versión 2.4 de dicha plataforma, operativa en UZ en 2013. De este modo, se ofrece una idea práctica de lo que se puede hacer con aprendizaje adaptativo de forma sencilla, eficiente y útil en el ámbito de un LMS como Moodle, sin añadir módulos adicionales o realizar desarrollos específicos. Se explicará el diseño pedagógico y la puesta en práctica de cada caso, más que los recursos digitales en sí mismos, (Gros, 2015) y se mostrarán los resultados, en términos de eficacia y eficiencia.

Los estudiantes eran alumnos pertenecientes a distintas titulaciones impartidas en la Escuela de Ingeniería y Arquitectura en UZ. El primer paso de adaptación empezaba por la titulación de procedencia de alumno, ya que las tres experiencias se desarrollaban dentro del mismo curso Moodle, pero cada estudiante solo accedía a la sección del mismo creada ad hoc según su pertenencia a una Titulación, Grupo de Docencia y Asignatura. La experiencia 1 se basó en el modelo en el que se desea producir una adaptación del proceso de aprendizaje a una variable inicial o característica del estudiante. Naturalmente esa variable debe estar definida en el modelo de usuario, indicando sus valores posibles. También debe decidirse el instrumento para determinar su valor en cada estudiante y, finalmente, debe diseñarse el proceso de aprendizaje específico asociado a cada valor de la variable.

Cada valor de esa variable inicial da acceso a un proceso de aprendizaje específico. Es muy frecuente utilizar como variables iniciales de adaptación el nivel de conocimiento o el estilo de aprendizaje, tipos de variables que exigen utilizar instrumentos para su diagnóstico, como cuestionarios de evaluación o test validados. En estos casos, el sistema inteligente muestra automáticamente el proceso de aprendizaje de cada estudiante una vez recogidas sus respuestas al instrumento de diagnóstico (Castelló, Lerís, Martínez y Sein- 
Echaluce, 2010). Otra alternativa consiste en utilizar instrumentos de decisión, permitiendo al estudiante que elija voluntariamente su participación en unos u otros procesos de aprendizaje. Esto significa que se admite la capacidad del estudiante para decidir lo más adecuado para su aprendizaje.

El diseño adaptativo de la experiencia 2 consiste en la navegación adaptativa del usuario por un itinerario de aprendizaje. Se trata de un modelo en el que el estudiante es guiado a través de las actividades del diseño instruccional, adaptándose a su propio ritmo. En definitiva, cada estudiante recorre el mismo itinerario de aprendizaje, pero sus propios pasos son los que van abriendo camino.

En este caso es necesario definir dos variables que controlan la navegación a través de los recursos: una variable temporal y otra de finalización de actividades. La variable temporal controla los momentos instruccionales; por ejemplo, para concordar los intervalos temporales presenciales con los no presenciales. La variable de finalización de actividades permite que el sistema despliegue automáticamente las siguientes actividades tras la conclusión de las previas; por tanto, adapta la navegación a los pasos dados por el estudiante. En resumen, el modelo utilizado consiste en una navegación tutelada a través de los contenidos, de modo que son las acciones de cada estudiante las que determinan la accesibilidad a los siguientes contenidos.

En la experiencia 3, además de la navegación adaptativa del usuario, se añade la diversificación del itinerario de aprendizaje. Es decir, los logros del estudiante determinan su acceso a ciertas partes del diseño instruccional. En este modelo es necesario determinar puntos clave en los que abrir caminos alternativos y bajo qué condiciones (Sein-Echaluce, Lerís y Fidalgo, 2011).

Además de la variable de tipo temporal y la de finalización de actividades presentes en la experiencia 2, se añaden variables de calificación. Estas controlan los caminos alternativos del diseño instruccional, que son sugeridos $\mathrm{u}$ obligatorios para que el estudiante avance en su proceso personal de aprendizaje.

Los resultados de aprendizaje en las tres experiencias en línea fueron evaluados a través del examen final de cada asignatura y de las actividades calificables incluidas en cada caso. Estas últimas, según el grupo de docencia, suponían hasta 1 o 2 puntos (sobre 10) de la calificación total de la asignatura. Por último, y una vez que el estudiante concluía el trabajo en línea, completaba un cuestionario multidimensional tipo SEEQ, acrónimo procedente de Student Evaluation of Educational Quality, (Marsh y Roche, 1997). Las preguntas del cuestionario SEEQ, formado por ítems valorados en una escala Likert de 1 a 5 , fueron adaptadas a cada uno de los tres contextos formativos y permitían conocer la calidad educativa percibida por el alumnado (Roda, López-Jaquero y Montero, 2015).

A continuación se describe cada caso y las 
características específicas del respectivo experimentado.

escenario de aprendizaje en el que se ha

\section{Descripción y resultados de los tres casos prácticos}

\subsection{Primera experiencia educativa. Adaptación del proceso de aprendizaje a una variable inicial}

La experiencia se llevó a cabo en la asignatura de Matemáticas II (Ecuaciones Diferenciales) de Ingeniería Técnica Industrial, especialidad Mecánica, del plan de estudios en extinción. La propuesta docente se realizó teniendo en

\subsubsection{Descripción}

Todos los estudiantes, participaran o no en la experiencia, disponían de una serie de ficheros de información con el contenido total de la asignatura, que les permitía prepararse de forma autónoma, para superar el examen global de la asignatura. Se diseñó un método alternativo de aprendizaje al que solo accedían aquellos estudiantes que así lo decidieran.

En efecto, dada la madurez de los estudiantes matriculados, todos ellos de segunda matrícula o más en la asignatura, con más de tres años de formación universitaria $y$, posiblemente, con diversas circunstancias formativas y personales, se optó por incluir un instrumento de decisión (consulta) en el que señalaran voluntariamente su participación en un proceso de aprendizaje especialmente diseñado. Se utilizó un instrumento de decisión para determinar el valor de la cuenta que se trataba de una asignatura sin docencia presencial en el curso 2012-2013. El número de matriculados, potenciales usuarios de la experiencia, era de veintitrés estudiantes.

variable de adaptación inicial. En la figura 2 se muestra el esquema de la adaptación.

El diseño adaptativo de esta experiencia se basó en el modelo en el que se desea producir una adaptación del proceso de aprendizaje a una variable inicial asociada al estudiante, que, en este caso, era su decisión de participar o no en dicho proceso. Si la respuesta a la consulta era afirmativa, el estudiante podía acceder a dos actividades -tareas- que se incorporaron a Moodle. La finalización de ambas tareas abría, a su vez, una encuesta de evaluación de la calidad educativa, tal y como se esquematiza en la figura 2.

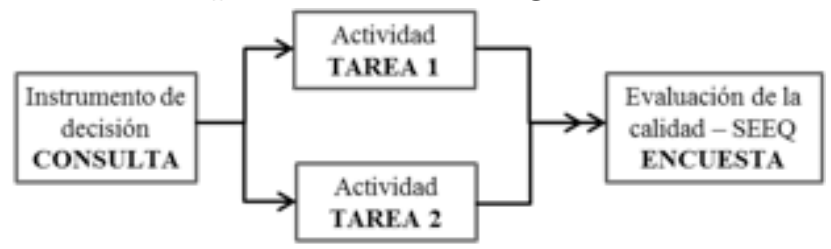

Figura 2. Flujo de aprendizaje de la parte adaptativa de la experiencia 


\subsubsection{Resultados}

Del total de veintitrés estudiantes en el proceso propuesto; datos que parecen matriculados, tan sólo nueve se incorporaron señalar que el hecho de haber participado a las actividades involucradas en el proceso de aprendizaje adaptativo, de los cuales seis entregaron la primera de las tareas y ocho la segunda.

La evaluación de las tareas se realizó de forma que la resolución correcta del $70 \%$ de las cuestiones planteadas en la tarea 1 y del $60 \%$ en la tarea 2, suponía dos puntos extra a sumar a la calificación del examen final. La puntuación media de los seis estudiantes que realizaron la primera tarea fue de 8 puntos sobre 10 (uno con 10 , dos con 9 , uno con 8 , uno con 7 y uno con 5); mientras que, en la segunda tarea, los ocho alumnos que la resolvieron obtuvieron una media de 6'75 puntos sobre 10 (dos con 10, tres con 8, dos con 4 y uno con 2).

El impacto puede medirse a través del examen de la asignatura, al que se presentaron nueve alumnos, de los que ocho habían participado está ligado a la presentación al examen final. Seis, de esos ocho participantes, superaron la asignatura; mientras que el único alumno presentado, que no participó en la experiencia, no aprobó la asignatura.

Debido al reducido número de estudiantes que cumplimentaron el cuestionario SEEQ, tan solo se indica una valoración cualitativa del juicio emitido por los estudiantes. Señalaron que la actividad online les había resultado más atractiva que las clases tradicionales y, además, les había permitido estudiar a su ritmo, teniendo la sensación de que el tiempo empleado en el estudio de la asignatura era menor y mejor empleado. También indicaron algunos aspectos de mejora, sobre todo los referentes a la retroalimentación en el caso de respuestas erróneas y la necesidad de automatizar algunos de los procesos desarrollados.

\subsection{Segunda experiencia educativa. Navegación adaptativa del usuario por un itinerario de aprendizaje}

La segunda experiencia se realizó en la del curso 2012-13. Todos los alumnos asignatura Matemáticas I de primer curso de los grados en Ingeniería Mecánica y en Tecnologías Industriales. Se dirigió a los veinticinco estudiantes matriculados en el participaron en la experiencia y todos tenían como característica común el hecho de haber cursado, sin éxito, la misma asignatura en anteriores cursos académicos. grupo de docencia del segundo cuatrimestre 


\subsubsection{Descripción}

La experiencia consistió en diseñar y realizar el aprendizaje de una unidad didáctica (los números complejos) en un entorno semipresencial, desarrollándose la actividad presencial en la clase y en las tutorías, mientras la no presencial se realizaba en Moodle.

El diseño adaptativo de esta experiencia se ocupaba de que la navegación por los recursos y herramientas se acomodara a las acciones del usuario. La navegación adaptativa se fundamentó en variables de finalización de las actividades. El diseño instruccional estaba formado por cinco subunidades, cuatro de ellas con idéntico flujo de aprendizaje (figura $3)$.

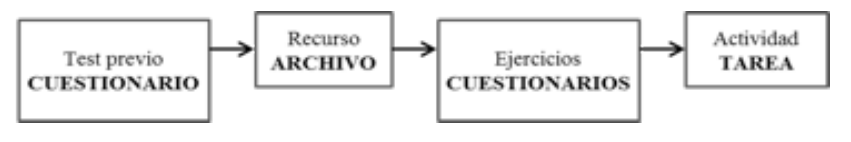

Figura 3. Flujo de aprendizaje común a cuatro unidades de la experiencia 2.

Como puede verse en la figura 3 , se comenzaba con la realización del test previo, o cuestionario de opción múltiple de conocimientos preliminares (sin trascendencia en la calificación final), que daba paso al recurso, un archivo con la información relativa al correspondiente apartado de la unidad. Una vez leído, el estudiante podía acceder a los ejercicios, cuestionarios (con efecto en la calificación final) para apoyar la comprensión de los conceptos. Finalmente, una vez contestados los cuestionarios, y sin tener en cuenta la calificación obtenida, ya podía realizar la última actividad de resolución de problemas abiertos.

La quinta subunidad de aprendizaje se refería al desarrollo histórico y contaba con un documento-resumen de cómo se fueron incorporando los números complejos al conocimiento matemático. Como se ve en la figura 4, su lectura llevaba a que el estudiante eligiera (consulta) el matemático sobre el que versaría la tarea de analizar sus aportaciones al proceso constructivo de este conjunto numérico. Una vez elegido, se abría la correspondiente tarea evaluable.

El modelo utilizado consiste, pues, en una navegación tutelada a través de los contenidos, de modo que son las acciones de cada estudiante las que determinan la accesibilidad a las siguientes actividades.

Como condiciones de navegación para los estudiantes en esta experiencia, se establecieron las de secuenciación de contenidos (tenían que seguir el flujo de la figura 3) y las de temporalidad (las actividades de una misma unidad debían realizarse en un intervalo temporal fijo), restricciones todas ellas admitidas en la versión de Moodle utilizada.

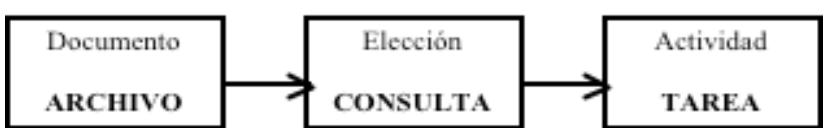

Figura 4. Flujo de aprendizaje de la quinta unidad la experiencia 2. 


\subsubsection{Resultados}

Respecto a la participación de los veinticinco estudiantes, hay que señalar que los cuestionarios de las cuatro subunidades los realizaron la práctica totalidad de los alumnos (la primera, 24 respuestas; la segunda, también 24; la tercera, 25 y la cuarta, 24); mientras que las tareas tuvieron una respuesta en torno al $75 \%$ (las tareas uno, dos y tres, 19 realizaciones y la cuarta tarea, 15).

Las calificaciones obtenidas en los cuestionarios y en las tareas evaluables suponían hasta 10 puntos en la nota final de la asignatura (sobre 100). La calificación media del grupo en esta experiencia fue de 6'95 sobre 10 puntos. Solo cuatro alumnos obtuvieron calificaciones inferiores a 5 (la más baja fue 3'89), doce en el intervalo $[5,7)$; ocho en el $[7,9)$ y uno con una calificación superior a 9 .

La eficacia de la experiencia queda avalada por los siguientes datos. Por un lado, el rendimiento fue altamente satisfactorio; ya que, entre las convocatorias de junio y de septiembre, aprobaron veinticuatro de los veinticinco alumnos matriculados (el alumno no aprobado no se había presentado al examen). Por otro lado, el éxito de la experiencia fue verificado a través de su repercusión en la calificación obtenida en la pregunta de números complejos incluida en el examen final. Para ello se propuso una pregunta semejante -solo cambiaban los datos empleados- a la realizada unos cursos antes en la convocatoria de septiembre. En el análisis estadístico efectuado sobre los datos de ambos exámenes, y tras las pertinentes comprobaciones previas, se realizó el contraste de la t de Student para muestras independientes. Los resultados del contraste indican que hay una diferencia estadísticamente significativa entre la puntuación media obtenida por los estudiantes de esta experiencia adaptativa, 5'73 puntos, y la conseguida por los estudiantes que no la habían realizado, 2'92 puntos.

Los alumnos participantes valoraron positivamente la experiencia en los distintos aspectos abordados en el cuestionario SEEQ, como puede verse en la figura 5. Además las respuestas a las preguntas abiertas señalan un alto grado de satisfacción con el diseño bien por haber podido trabajar fuera del aula y por estar adaptado a su ritmo, bien por la progresiva dificultad de los contenidos.

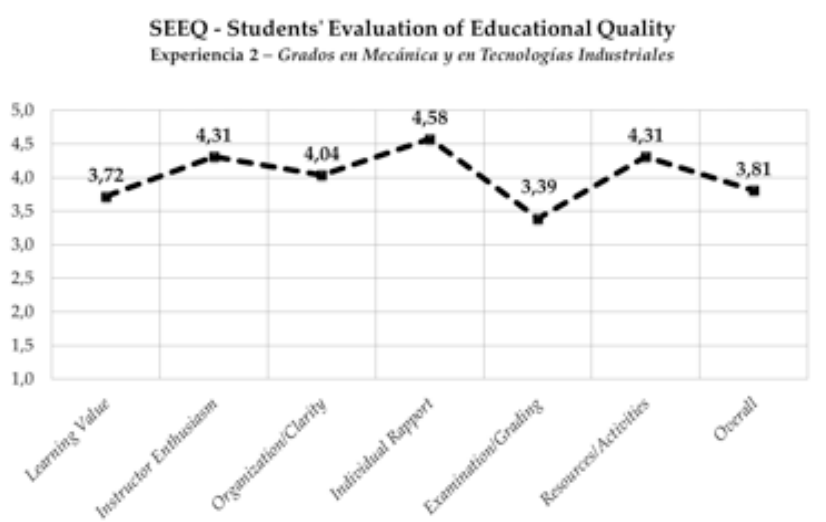




\subsection{Tercera experiencia educativa. Adaptación del itinerario de aprendizaje}

La tercera experiencia se llevó a cabo en la asignatura Matemáticas II del segundo cuatrimestre de primer curso del grado en Ingeniería Eléctrica. El diseño instruccional contemplaba la realización completamente online de las dos últimas partes de una unidad de aprendizaje, cuyo contenido no se explicó

\subsubsection{Descripción}

Esta experiencia, además de la adaptación de la navegación coincidente con la de la anterior, añadía la diversificación de itinerarios y algunos mensajes adaptativos que guiaran al estudiante en su personal camino. Así pues, el modelo de usuario contenía variables de finalización de las actividades, en las que se fundamentó la navegación adaptativa, y variables de calificación en las que se basaron los caminos alternativos.

La estructura de la unidad didáctica online constaba de un camino de aprendizaje, común para todos los alumnos, que incluía una bifurcación intermedia solo disponible en aquellos casos en que los resultados previos aconsejaran un refuerzo. Este camino común de objetivos mínimos se prolongaba con unas actividades complementarias, destinadas a aquellos estudiantes que hubieran alcanzado el dominio necesario de los contenidos mínimos. La navegación (restricciones de acceso) se configuró para que se adaptara al en clase, pero sí que formó parte de la materia del examen de la asignatura. La experiencia estaba dirigida a los estudiantes matriculados en los dos grupos de docencia, en total 102 alumnos, que no tenían ninguna de las características especiales de los participantes en las dos experiencias anteriores.

ritmo del estudiante. Se habilitaron textos (etiquetas), que cambiaban según las acciones del estudiante, y se utilizaron actividades Moodle (cuestionarios y lecciones) que, en sí mismas, se adaptaban a las acciones o a la voluntad del estudiante.

En la figura 6 se muestra el flujo de aprendizaje de esta tercera experiencia. La introducción y los dos bloques siguientes constituyen el itinerario común, en el que únicamente está adaptada la navegación al ritmo de trabajo del estudiante. La actividad rotulada Refuerzo, junto con su etiqueta informativa publicada en Moodle, solo forma parte del camino de aprendizaje del estudiante con calificaciones inferiores al $40 \%$ en los test 1 a 3. Finalmente, las actividades bajo el título Complemento, solo son accesibles para los estudiantes que hayan alcanzado un nivel de conocimientos suficiente (la calificación de aprobado en el test 4).

Se debe señalar que las actividades, 
interactivas en sí mismas, realizadas en esta experiencia son:

- Los tres Test Interactivos con Pistas, tanto los dos iniciales de la introducción como el de refuerzo, estaban concebidos como medio activo para que el estudiante fuera construyendo su propio conocimiento con la ayuda de pistas. Las pistas se activaban únicamente si las respuestas del estudiante eran incorrectas.

- Las dos Lecciones, cuya misión era ofrecer ejemplos de los conceptos y procedimientos descritos en los archivos, permitían que fuera el propio estudiante el que decidiera qué información sobre los ejemplos incluidos quería ver y en qué momento.

\subsubsection{Resultados}

La participación en la experiencia superó globalmente el $50 \%$ de los estudiantes matriculados (la introducción la realizaron 57 alumnos; mientras que el primer test fue contestado por 55, el segundo por 54, el tercero por 53 y el cuarto por 52). La tarea final, que era opcional, fue completada por 17 alumnos, lo cual supone el $30 \%$ de los que comenzaron la experiencia; si bien fueron 29 estudiantes -más de la mitad de los que empezaron- los que llegaron a la consulta previa a dicha tarea. Todo el proceso era automático salvo esta última actividad, cuya revisión y calificación se realizó manualmente. La media de las calificaciones obtenidas

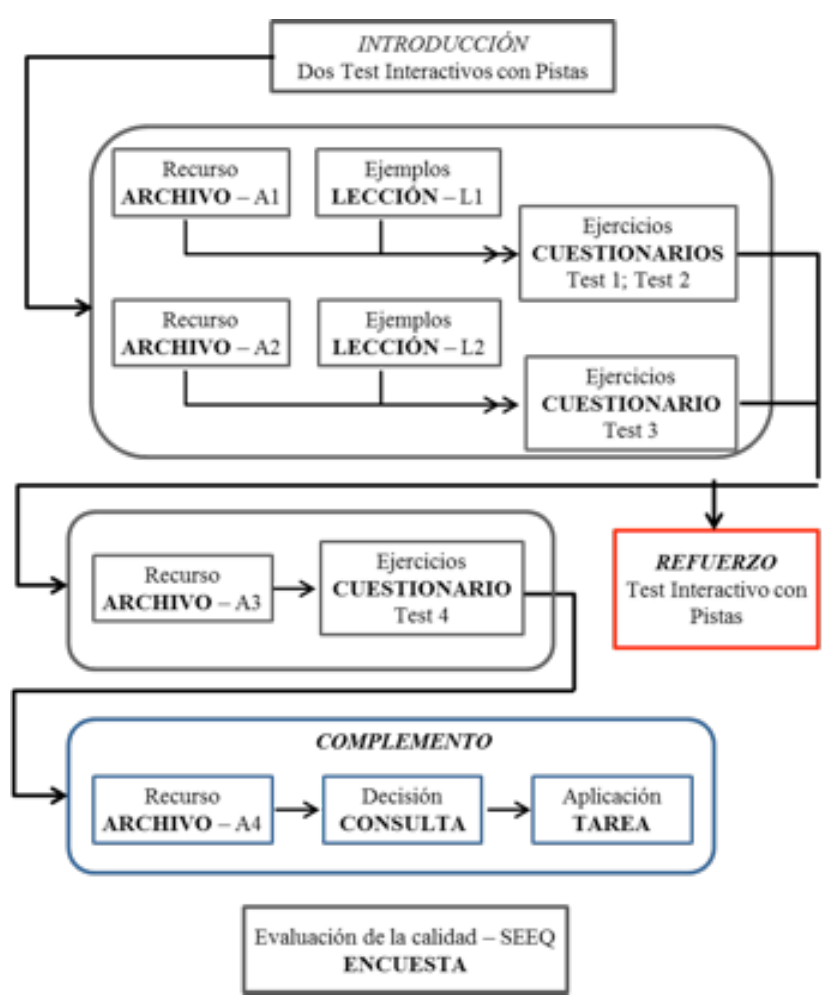

Figura 6. Flujo de aprendizaje de la experiencia 3.

en los test 1 a 4 (calificados sobre 5) más la de la tarea del bloque complementario (puntuación máxima 5) contribuían a la calificación final de la asignatura. Con ello se podía obtener hasta diez puntos extra dentro de la calificación total (sobre 100) de la asignatura. Además, los conceptos tratados en el curso online formaron parte del examen final, en el que se propuso un ejercicio cuyo peso fue del $20 \%$.

Las calificaciones medias, obtenidas en los distintos recursos utilizados en la experiencia, fueron muy satisfactorias. Empezando con los dos test iniciales con pistas (9'26 y 8'34 puntos sobre 10) y continuando con los 
cuatro test de desarrollo del tema (todos ellos evaluados sobre 5 puntos obtuvieron de media 3'54, 3'50, 4'24 y 3'58). La media de los cuatro test fue de 3'60 puntos. La nota media de la tarea opcional alcanzó los 3’59 sobre 5 puntos.

Además, hay que señalar los buenos resultados en el examen final de la asignatura. En efecto, por un lado, el $80 \%$ de los estudiantes que participaron en la experiencia se presentaron al examen final de la convocatoria de junio de 2013. Y, por otro, la quinta pregunta de dicho examen -la correspondiente a los contenidos tratados en la experiencia- obtuvo mejor media de calificación que las demás. Las mencionadas calificaciones, sobre 10 , fueron: la primera pregunta con 4'5 puntos, la segunda $1^{\prime} 7$ puntos, la tercera 5 puntos, la cuarta 3'8, la quinta pregunta 5'2 puntos y la sexta 1'1.

\section{Conclusiones}

Para constatar el resultado del trabajo desarrollado, nos hemos centrado en valorar si ha mejorado el aprendizaje, en términos de eficiencia y eficacia de la enseñanza, y si ha sido satisfactorio para los estudiantes.

Consideramos que una práctica educativa es eficiente si resulta aceptable el esfuerzo del profesor (creador y tutor) en relación a los logros. En las tres experiencias ha sido clave la reutilización de los objetos de aprendizaje, ya que ha supuesto una ventaja en términos de esfuerzo del profesorado
Respecto a la opinión de los alumnos, manifestada a través del mencionado cuestionario SEEQ, en la figura 7 se aprecia el nivel de satisfacción en los aspectos recogidos en la encuesta. Asimismo, las respuestas abiertas muestran un alto grado de aceptación de la experiencia, tanto por haberla podido realizar a su ritmo y en el momento y lugar deseado, como por la cantidad de ejemplos desarrollados, que, a su juicio, les facilitaban el aprendizaje.

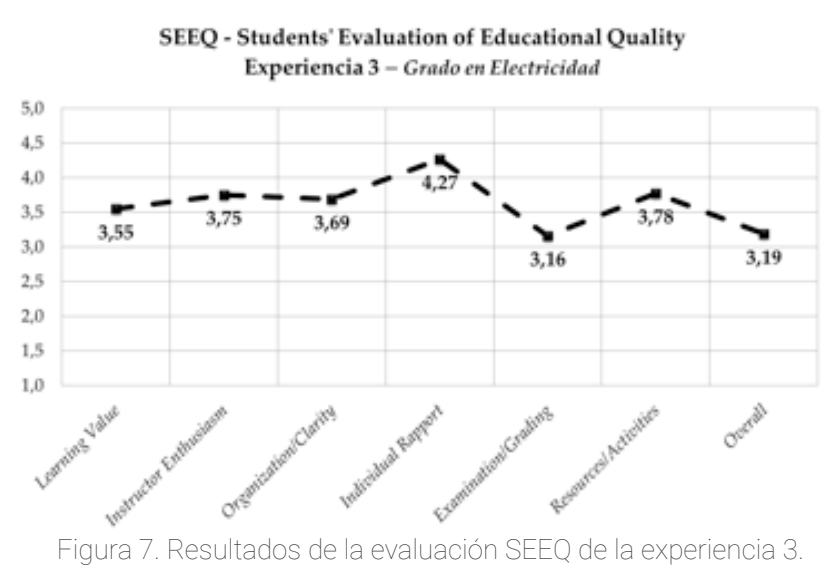

y ha permitido dirigirlo a adaptar dichos objetos al nuevo entorno de Moodle y al diseño e implementación de los caminos de aprendizaje.

La eficacia de la enseñanza ha sido valorada mediante el ya clásico criterio del aprendizaje logrado por el estudiante. El impacto en la eficacia de la enseñanza, derivado de las tasas de éxito (aprobados/presentados) y rendimiento (aprobados/matriculados) de la asignatura, ha sido evidente a la luz de los datos mostrados. Restringiéndonos en cada 
caso a los aspectos o contenidos trabajados de forma online, con su correspondiente presencia de ellos en el examen final, podemos afirmar que la tasa de éxito ha sido igual o superior al $75 \%$ y la de rendimiento igual o superior al $66 \%$.

En la valoración de cada experiencia, que los estudiantes realizaron a través de un cuestionario multidimensional tipo SEEQ, las puntuaciones medias siguen un mismo patrón en las tres experiencias. Todas las dimensiones evaluadas alcanzan valores superiores al intermedio en la escala Likert de 1 a 5 .

Para finalizar este trabajo y como conclusión de él, entendemos que los tres modelos de aprendizaje adaptativo, basados en Moodle, son útiles, mejoran la enseñanza académica y son fácilmente aplicables en las clases reales. Además, la implementación se ha realizado sobre una instalación estándar de Moodle, no ha sido necesario añadir módulos adicionales ni realizar desarrollos de software. Esos son los puntos clave que nos permiten afirmar, no solo la sostenibilidad o continuidad de nuestro trabajo, sino también su idoneidad para ser transferidos a cualquier contexto formativo. Por último, en las experiencias realizadas, se han observado las limitaciones adaptativas de la versión de Moodle utilizada (la 2.4), que, en alguna ocasión, exigieron modificar los diseños instruccionales iniciales.

\section{Agradecimientos}

Este trabajo ha sido apoyado por el Gobierno de Aragón, el Fondo Social Europeo y la Universidad de Zaragoza.

Queremos agradecer a la profesora Blanca Bellostas Solanilla, responsable de la realización de la primera de las experiencias expuestas en el presente artículo, por su dedicación y generosidad; así como la valiosa aportación de la profesora Belén SánchezValverde García al análisis estadístico de los datos del segundo de los casos prácticos desarrollados.

\section{Referencias}

Amo, D., Casany, M. Alier, M. (2014). Designs. En Proceedings of the Workshop on Approaches for quality in pedagogical and Applications of Semantic Web Technologies design fundamentals in moocs. Education in for Educational Adaptive Hypermedia, 354the Knowledge Society (EKS), 15(1), 70-89. 358.

Berlanga, A. y García-Peñalvo, F. J. (2004). Berlanga, A. y García-Peñalvo, F. J. (2005). A Proposal to Define Adaptive Learning IMS LD reusable elements for adaptive 
learning designs. Journal of Interactive Media in Education, 2005(11), Art-12. http:// dx.doi.org/10.5334/2005-11

Berlanga, A. y García-Peñalvo, F. J. (2008). Learning Design in Adaptive Educational Hypermedia Systems. J. UCS, 14(22), 36273647.

Bontchev, B. y Vassileva, D. (2012). Courseware Adaptation to Learning Styles and Knowledge Level. En E-Learning - Engineering, On-Job Training and Interactive Teaching (Edited by Dr. Sergio Kofuji., pp. 3-22). InTech. http://dx.doi. org $/ 10.5772 / 29340$

Bra, P., Smits, D., Sluijs, K., Cristea, A., Foss, J., Glahn, C. y Steiner, C. (2013). GRAPPLE: Learning Management Systems Meet Adaptive Learning Environments. En A. Peña-Ayala (Ed.), Intelligent and Adaptive Educational-Learning Systems (Vol. 17, pp. 133-160). Springer Berlin Heidelberg.

Brusilovsky, P., Knapp, J. y Gamper, J. (2006). Supporting teachers as content authors in intelligent educational systems. International Journal Knowledge and Learning, 2 (3/4), 191-215. http://dx.doi. org/10.1504/IJKL.2006.010992

Burgos, D., Tattersall, C., Dougiamas, M., Daniel, S. J., Vázquez Cano, E. y Gisbert, Vogten, H. y Koper, R. (2007). A First Step M. (2015). The Future of MOOCs: Adaptive Mapping IMS Learning Design and Moodle. Learning or Business Model? RUSC. J. UCS, 13(7), 924-931.
Calderero Hernández, J. F., Aguirre Ocaña, A. M., Castellanos Sánchez, A., Peris Sirvent, R. M. y Perochena González, P. (2014). Una nueva aproximación al concepto de educación personalizada y su relación con las TIC. Education in the Knowledge Society (EKS), 15(2), 131-151.

Castelló, J., Lerís, D., Martínez, V. y Sein-Echaluce, M. L. (2010). Personalized Learning on the Moodle Platform using the CICEI Conditionals: Support Course in Mathematics. INTED2010 Proceedings International Technology, Education and Development. Publisher: IATED, Valencia, Spain.

CICEI Centro de Innovación para la Sociedad de la Información. Actividades condicionales en Moodle.

Conlan, O., Wade, V., Gargan, M., Hockemeyer, C. y Albert, D. (2002). An architecture for integrating adaptive hypermedia services with open learning S. (eds.) Proc. of ED-MEDIA'2002 - World Conference on Educational Multimedia, Hypermedia and Telecommunications, (Denver, CO, June 24-29, 2002), AACE, 344350.

Universities and Knowledge Society Journal, 
12(1), 64. http://dx.doi.org/10.7238/rusc. to break the current limitations of MOOC v12i1.2475

model. Journal of Universal Computer Science, 21, 712-734.

Despotović-Zrakić, M., Marković, A.,

Bogdanović, Z., Barać, D. y Krčo, S. (2012).

García-Peñalvo, F. J. y Seoane-Pardo, A. Providing Adaptivity in Moodle LMS Courses. Journal of Educational Technology 8 Society, 15(1), 326-338. M. (2015). Una revisión actualizada del concepto de eLearning. Décimo Aniversario. Education in the Knowledge Society (EKS), 16(1), 119-144. http://dx.doi.org/10.14201/

Fidalgo, Á., Sein-Echaluce, M. L., Lerís, D. eks2015161119144

y Castañeda, O. (2013). Teaching Innova Project: the Incorporation of Adaptable Outcomes in Order to Grade Training Adaptability. J. UCS, 19(11), 1500-1521.

Gros, B. (2015). La caída de los muros del conocimiento en la sociedad digital y las pedagogías emergentes. Education in the Knowledge Society (EKS), 16(1), 58-68.

Fidalgo-Blanco, Á., García-Peñalvo, F. J. y http://dx.doi.org/10.14201/eks20151615868 Sein-Echaluce, M. (2013). A methodology proposal for developing adaptive Johnson, L., Adams, S., Cummins, M. y cMOOC. En Proceedings of the First Estrada, V. (2012). Technology Outlook for International Conference on Technological STEM+ Education 2012-201\%: An NMC Ecosystem for Enhancing Multiculturality Horizon Report Sector Analysis.Austin, (pp. 553-558). ACM. http://dx.doi. Texas: The New Media Consortium. org $/ 10.1145 / 2536536.2536621$

Lerís, D. y Sein-Echaluce, M.L. (2011). La

Fidalgo-Blanco, A., García-Peñalvo, F. J., personalización del aprendizaje: un objetivo Sein-Echaluce, M. L. y Conde-González, M. del paradigma educativo centrado en el A. (2014). Learning content management aprendizaje. Arbor: Ciencia, pensamiento systems for the definition of adaptive learning y cultura, 187 (3), 123-134. http://dx.doi. environments. En Computers in Education org/10.3989/arbor.2011.iExtra_3 (SIIE), 2014 International Symposium on (pp. 105-110). IEEE. http://dx.doi.org/10.1109/ SIIE.2014.7017713

Marsh, H. W. y Roche, L. A. (1997). Making students' evaluations of teaching efectiveness effective. The critical issues of validity, bias

Fidalgo-Blanco, Á., Sein-Echaluce, M. L. y García-Peñalvo, F. J. (2015). Methodological Approach and Technological Framework and utility. American Psychologist, 52(11), 1187-1197. http://dx.doi.org/10.1037/0003066X.52.11.1187 
Miliband, D. (2006). Capítulo 1 "Choice

and Voice in Personalised Learning", Skinner, B. F. (1958). Teaching Machines. pp. 21-30, Schooling for tomorrow- Science, 128 (3330), 969-977. doi: http:// Personalising Education. http://dx.doi. dx.doi.org/10.1126/science.128.3330.969 org/10.1787/9789264036604-2-en

Sonwalkar, N. (2013). The first adaptive Rey-López, M., Brusilovsky, P., Meccawy, MOOC: A case study on pedagogy framework M., Díaz-Redondo, R., Fernández-Vilas, A. and scalable cloud Architecture-Part I. En y Ashman, H. (2008). Resolving the problem MOOCs Forum (Vol. 1, pp. 22-29). Mary of intelligent learning content in learning Ann Liebert, Inc. 140 Huguenot Street, 3rd management systems. International Journal Floor New Rochelle, NY 10801 USA. on E-Learning, $7(3), 363-381$.

Tiarnaigh, M. (2005). An integration of Roda, C., López-Jaquero, V. y Montero, Moodle (Modular Object-Oriented Dynamic F. (2015). Hacia la caracterización de la Learning Environment) with an AHS calidad de interacción. Proceedings of the (Adaptive Hypermedia System). Final Year XVI International Conference on Human Project.

Computer Interaction, 429-437. Septiembre de 2015, Vilanova i la Geltrú, Barcelona, Vassileva, D. (2010). Storyboard Design for Spain.

Adaptive E-learning Based on Learning Styles (pp. 11-12). Second International Conference

Sein-Echaluce, M. L., Lerís, D. y Fidalgo, S3T, Varna, Bulgaria.

A. (2011). Diseño instruccional adaptativo de cursos online en Ingeniería. Promotion and Innovation with New Technologies in Engineering Education (FINTDI) - IEEE Conferences, 1-8, 5-6 May 2011. http:// dx.doi.org/10.1109/FINTDI.2011.5945972

Vélez, J., Baldiris, S., Nassiff, S. y Fabregat, R. (2008). Generación de cursos virtuales adaptativos basados en SCORM e IMS-LD. Avances en Sistemas e Informática, 5(3), 4959. 